\title{
Humanlike Behavior Model with Probabilistic Intention
}

\author{
Naoki Ibe, Gakuto Masuyama, Atsushi Yamashita, Hajime Asama \\ The University of Tokyo*
}
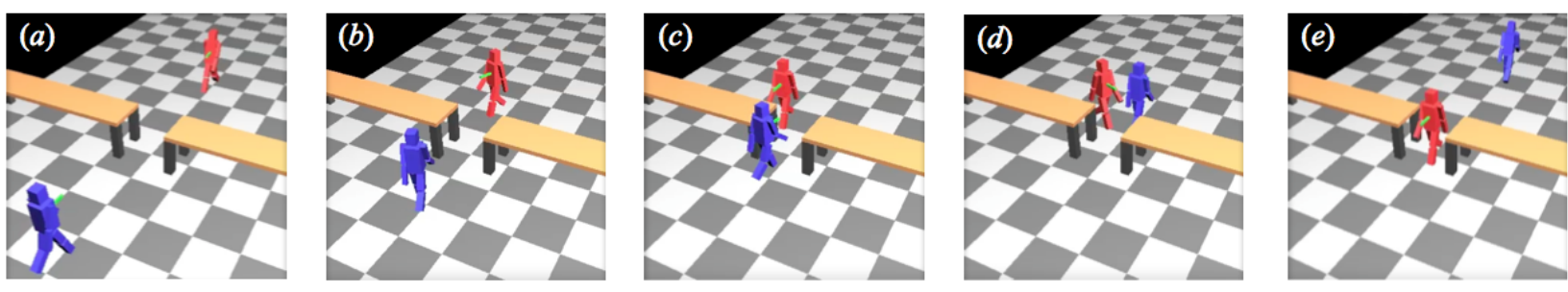

Figure 1: Give-way behavior at a narrow passage. (a), (b): Pedestrian A (blue) cannot decide to whether give-way or not because the intention of $\mathrm{B}(r e d)$ is unclear. Hence "hesitation" of $\mathrm{A}$ is produced, which means A alternates between $(a)$ directing to the passage and $(b)$ giving-way. (c): A enters the passage because it is estimated that $\mathrm{B}$ gives way. (d), (e): B enters the passage after $\mathrm{A}$ has passed.

\section{Introduction}

Animating the motion of a realistic crowd of pedestrians has been an important goal in the field of computer graphics, video games and movies. Pedestrians in the real world seem to consider not only collision avoidance but also interaction with other pedestrians. Thus, it is required to develop pedestrian models that produce humanlike behavior which includes social interactions such as giving-way in order to simulate the realistic motion of pedestrians. Human behaviors and activities have been studied with various approaches. Social Force Model (SFM) describes each pedestrian's motion by virtual forces from its target point, other pedestrians and surrounding environment [Helbing and Molnár 1995]. Each individual motion is described by differential equations and calculated in parallel. SFM is one of the most suitable models to simulate the motion of pedestrians and there are several extensions to simulate pedestrian behaviors in large spaces by applying some rules to SFM (e.g., [Belechanoet al 2007]). However, these studies treated pedestrian intention as the internal state which consists of several discrete states though the human intention in the real world is continuous and ambiguous. As a result, humanlike behaviors could not be produced when the intention of other pedestrian is unclear.

In this paper, the pedestrian model that produces humanlike behaviors by considering probabilistic intention of pedestrians is demonstrated. The pedestrian intention is represented as a superposition of orthogonal states that correspond to behaviors such as givingway and directing to the goal. We also propose a new method to predict the pedestrian motion by the inverse calculation of SFM.

\section{Approach}

It must be necessary to take into account probabilistic intention of pedestrians and estimate other's intention by long-term prediction of other's motion, in order to realize humanlike behaviors.

Introduction of probabilistic intention In our model, SFM is adapted to calculate actual motion and our model of pedestrians is designed to switch their behavior by changing subgoal that corresponds to the target point in SFM. We introduce a mechanism to select a suitable subgoal according to the probabilistic intention of the pedestrian at the moment. To introduce the probabilistic intention, this research focused on the idea of quantum information theory. Pedestrian intention is represented by quantum state $|\psi\rangle$

\footnotetext{
*e-mail:\{ibe, masuyama, yamashita, asama\}@ robot.t.u-tokyo.ac.jp
}

called "intention vector." $|\psi\rangle$ is a superposition state as follows:

$$
|\psi\rangle=\sum_{i} c_{i}\left|s_{i}\right\rangle, \quad \sum_{i}\left|c_{i}\right|^{2}=1
$$

where $c_{i}$ are complex coefficients, and $\left|s_{i}\right\rangle$ are orthogonal states called "intention state" and they correspond to the behaviors that are mutually exclusive. $\left|c_{i}\right|^{2}$ represents occurrence probability of $\left|s_{i}\right\rangle$ at each step. Hence each behaviors is produced from each intention state according to the corresponding probability.

Estimation of other pedestrian intention Each pedestrian predicts the future route of other pedestrian by the inverse calculation of the SFM. Firstly, a pedestrian estimates the direction of other's subgoal from other's motion and surrounding environment by inverse calculation of SFM. Then, using the estimated direction of other's subgoal, future positions of other pedestrian is predicted sequentially by SFM. The point set of the future positions is considered as the future route. Finally, other's intention is estimated according to the evaluation value calculated along the future route from the function whose value depends on the distance between the future position at each moment and subgoals. Each pedestrian changes its intention according to the estimated intention of other pedestrian and produce the suitable behavior.

\section{Implementation and Future Work}

We applied the proposed model in the situation where there is a narrow passage between two obstacles. In the experiment, two pedestrians approached each side of the passage and attempted to pass to the other side. It was shown that one pedestrian generates give-way behavior and the other enters the passage (Figure 1). In addition, our model can describe "hesitation" of pedestrians that cannot be realized by conventional models. Currently, we extend the model to generate other humanlike behaviors and to be valid for various environment and intention.

\section{References}

Helbing, D., And Molnár, P. 1995. Social force model for pedestrian dynamics. Physical Review E 51, 4282-4286.

Pelechano, N., Allbeck, J. M., And Badler, N. I. 2007. Controlling individual agents in high-density crowd simulation. In Proceedings of the 2007 ACM SIGGRAPH/Eurographics Symposium on Computer Animation (SCA2007), 99-108. 